\title{
Proving Isolation Properties for Software Transactional Memory ${ }^{\star}$
}

\author{
Annette Bieniusa and Peter Thiemann \\ University of Freiburg, Germany
}

\begin{abstract}
An algorithm for Software Transactional Memory (STM) is correct if it guarantees a proclaimed degree of isolation between concurrently executing transactions. A correctness proof requires explicit modeling of the effects of transaction bodies and the non-deterministic scheduling of their operations.

We provide a formalization of an STM algorithm that is explicit about all aspects required for a correctness proof: effects of operations, nondeterminism, and modeling rollback. We prove that this algorithm is correct by showing that it implements opacity.
\end{abstract}

\section{Introduction}

Concurrent programming is notoriously difficult. Programs that rely on shared memory may exhibit race conditions or other strange effects caused by the subtleties of the underlying memory model. Traditionally, these defects are kept at bay through synchronization primitives like monitors, locks, and semaphores. However, these constructs complicate reasoning about programs considerably, not the least because they might give rise to deadlocks.

While an application aiming for ultimate performance has to tackle these obstacles and must rely on explicit reasoning with the synchronization primitives to construct a correctness proof, Software Transactional Memory (STM) is an alternative approach that gives high-level guarantees about the interaction of concurrent threads. In STM, read or write accesses to shared memory are only permitted inside a transaction, where a thread is guaranteed an isolation property which roughly states that it never sees an inconsistent memory snapshot.

The problems resulting from computations that observe inconsistencies in their memory snapshot are well-known. For example, in an interleaved execution of transactions $\mathrm{Tx} 1$ and $\mathrm{Tx} 2$,

Tx1: $\operatorname{read} \mathrm{X}$;

read $\mathrm{X}$

Tx2: $\quad$ write $X$; commit

the second read should return the same value as the first one (repeatable read) and, furthermore, transaction $\mathrm{Tx} 1$ must not be allowed to commit because its outcome may be based on an obsolete value of $\mathrm{X}$.

\footnotetext{
* This work was partially funded as part of the JCell project by the Federal Government of Germany under grant number 01IH08011.
} 
For another example, consider the interleaved execution

Tx1: $\quad$ read $\mathrm{X}$; commit

Tx2: write $\mathrm{X}$; abort;

where the read operation in Tx1 must not return the value written by Tx2 because it aborts instead of committing (phantom read). The first transaction Tx1 may commit unless there are inconsistencies involving other variables.

By avoiding observable inconsistencies, the semantics of transactional memory provides a comparatively simple model for concurrent programming. Instead of having the programmer associate several memory locations with a lock and requiring that the lock needs to be obtained before accessing any of these memory locations and released thereafter, accesses are grouped together in a transaction that runs at a proclaimed level of isolation. To prevent conflicting accesses, each STM algorithm has a built-in contention strategy that detects and resolves conflicts like the ones exhibited in the examples. The main question is thus what kind of isolation does an STM algorithm provide.

Prior work on the semantics of transactions [112 focused primarily on weak atomicity, which is important for hybrid applications (for example, an application that includes legacy code using locking as well as new transactional code) because it helps to study the interaction of transactional and non-transactional memory accesses. However, these formalizations do not account for the phenomena due to the interleaved execution of transactions. For example, in state-of-the-art algorithms like TL2 [4], threads may get stuck even when a fair scheduling of threads is provided because they are repeatedly forced to abort by other transactions' successful commits.

To illustrate the mechanism underlying the aborts, this paper pursues an approach that abstracts program execution by traces of memory accesses and transaction control operations. To this end, we define a monadic lambda calculus with threads and transactions, $\Lambda_{S T M}$. Similar to approaches in research on isolation levels of database transactions, each memory access is modeled by an effect on the global store. By modeling an TM algorithm similar to TL2, the system is shown to implement the isolation level of opacity.

We claim the following contributions:

1. A formalization of a semantics of transactional memory that is suitable for proving properties of a TM implementation.

A high-level semantics abstracts so many details that properties of the implementation become trivially evident [8]. A low-level semantics provides so many details that formal proof of its properties is no longer tractable. An example is pseudocode for an implementation. Our semantics keeps in the middle ground. It explicitly models the non-deterministic interleaving of the operations in each thread including operations in aborting transactions. However, it does not model implementation details like the construction of memory snapshots or the implementation of locks.

2. We prove that our semantics implements opacity [7], that is, all execution traces in our semantics are equivalent to serial execution traces, where the execution of critical regions (i.e., transaction bodies) is non-interleaved. 
Overview. Section 2 starts with examples that illustrate the basic ideas. Then, section 3 presents the formalization of the system. Based on the operational semantics, section 4 continues with the characteristics of the model and shows the serializability of traces. The paper concludes with related work in section 5 and concludes in section 6 .

\section{Execution Traces}

\subsection{Successful Commits}

If all transactions in a program run commit successfully at the highest isolation level, then the execution trace can be turned into a serializable one by adapting the scheduler's decisions. For an example, consider a trace where the scheduling interleaves two transactions $t_{1}$ and $t_{2}$ that read and write disjoint variables $x \neq y$ (at marks the start of a transaction and $c o(x)$ its commit which writes variable $x)$ :

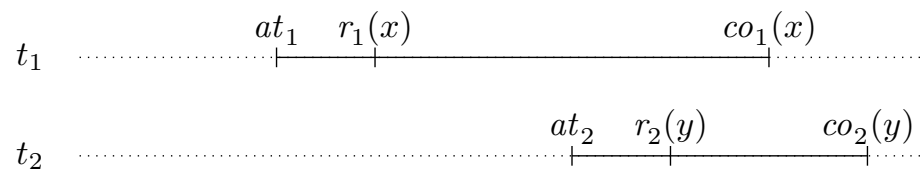

For this trace, there are two equivalent serial traces:

$$
\begin{aligned}
& t_{1} \quad \ldots \ldots \ldots \ldots \ldots \ldots
\end{aligned}
$$

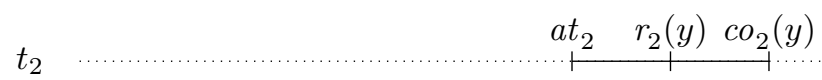

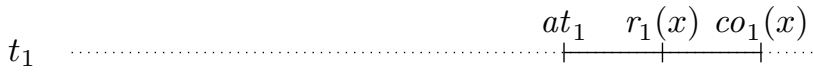

$$
\begin{aligned}
& t_{2} \quad \ldots \ldots \ldots \ldots
\end{aligned}
$$

Both traces correspond to evaluations of the program to the same final store. During the evaluation, each transaction conceptually operates on its own memory snapshot, taken at the beginning of the transaction. As both transactions were able to finish successfully, their read and write sets cannot have elements in common, and all their operations are independent. Hence both serial traces are equivalent to the original one.

\section{$2.2 \quad$ Read Conflicts}

A read conflict occurs if one transaction commits a write operation to a variable that another transaction is just about to read. In that case, the reading transaction must not proceed because its snapshot is no longer consistent with the current store. Thus, the semantics forces the second transaction to abort. 
As an example, consider the following trace:

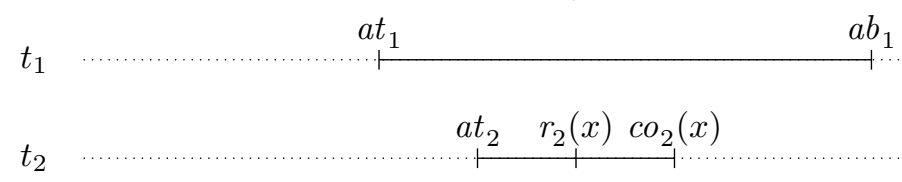

The trace illustrates a transaction $t_{1}$ which has to abort (indicated by $a b$ ) because of a read conflict on variable $x$ (it does not produce a read effect because the read operation is never permitted, as explained above). Nevertheless, there is an equivalent serial trace:

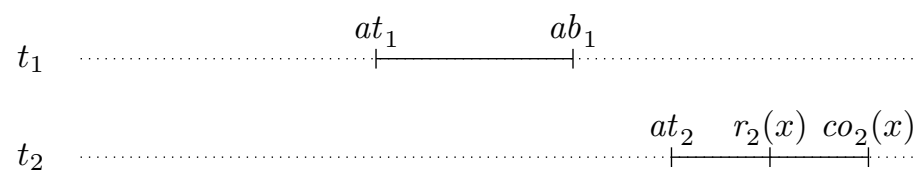

\subsection{Snapshot Isolation}

The next trace gives an example of a non-serializable execution:

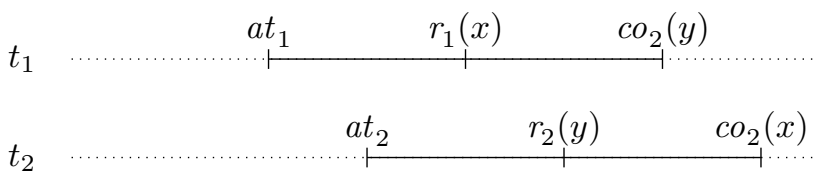

The transactions in this example do not have write or read conflicts as they update different memory locations. Yet, the memory locations that one transaction read are updated by the other. This effect is a write skew anomaly [2]. The trace is not serializable because a read operation is supposed to return the last value written to a variable. Hence, in a serial trace the latter operation would yield the value written by the first one.

Algorithms that allow traces with write skew anomalies implement a weaker isolation level called snapshot isolation. It requires that all transactions operate on reads that belong to one consistent memory snapshot. Updates that are committed between the begin of a transaction and its commit lead to conflicts only if the current transaction is about to update the same locations.

\subsection{Semantics of Haskell's orElse}

The STM library for Haskell [8], which is shipped with GHC, provides a construct $m_{1}$ 'orElse' $m_{2}$. Its semantics specifies that if there is a failure during the execution of the or branch $m_{1}$ (because of a failed read due to a conflict with a concurrently running transaction or because of a retry instruction issued by the programmer), the write operations of the or branch are discarded, and else branch $m_{2}$ is executed instead.

Although the semantics of Haskell's STM does not permit write skew anomalies, it provides the programmer with information about conflicting memory accesses at run time. If the programmer exploits this information, the semantics 


\begin{aligned} &$x \in \operatorname{Var} l \in \operatorname{Ref} \\ & v \in \operatorname{Val}::= l|\mathrm{tt}| \mathrm{ff}|()| \lambda x . e \\ & e \in \operatorname{Exp}::= v|x|$ ee $\mid$ if $e$ e e $\mid$ return $e \mid e \gg e \\ & \mid$ spawn $e \mid$ atomic $e \mid\left(e, W_{i}, R_{i}, i, e, \mathcal{H}\right) \\ & \mid$ newref $e \mid$ readref $e \mid$ writeref $e e \\ &$\hline\end{aligned}

Fig. 1. Syntax of $\Lambda_{S T M}$. Gray expressions arise only during evaluation.

of a program depends crucially on the scheduling of the threads. As the else branch is never entered in a serial execution (unless there is an explicition invocation of retry), Haskell STM actually implements an isolation level which is less restrictive than opacity but more restrictive than snapshot isolation.

This paper concentrates on serializability and opacity. It does not consider weaker isolation modes like snapshot isolation or Haskell's orElse construct.

\section{Formalization of Transactions}

This section formalizes an STM with lazy update, where all write operations are delayed till the commit operation. The formalization is based on a monadic call-by-value lambda calculus with references, threads, and transactions.

\subsection{Syntax}

Figure 1 contains the syntax of $\Lambda_{S T M}$. A value is either a reference, a boolean, the unit constant, or a function. Expressions comprise these values, variables, function application, conditional, monadic return and bind, spawning of threads, transactions, transactions in progress (an intermediate expression not arising in source programs), and the usual operations on references. The expression $e_{1} ; e_{2}$ abbreviates $\left(\lambda x . e_{2}\right) e_{1}$ where $x$ does not appear free in $e_{2}$ and $e[v / x]$ denotes the capture-avoiding substitution of $x$ by $v$ in $e$.

Figure 2 defines the type system for $\Lambda_{S T M}$. The type language consists of the types of the simply typed lambda calculus with base types boolean and unit, a reference type $\mathrm{R} \tau$ for references pointing to values of type $\tau$, function types, and a monadic type $\mu \tau$ for a monad returning values of type $\tau$. There is a choice of two monads, IO for general monadic operations and STM for operations inside a transaction.

The typing judgment contains two environments: $\Sigma$ tracks the type of memory locations, and $\Gamma$ tracks the type of variables. There is a second, heap typing judgment that relates the type of each memory location to the (closed) value stored in it. The typing rules are syntax-directed and mostly standard.

\subsection{Operational Semantics}

Figure 3 introduces some further definitions for the operational semantics. A program state $\mathcal{H}, \mathcal{P}$ is a pair consisting of a heap and a thread pool. A thread 


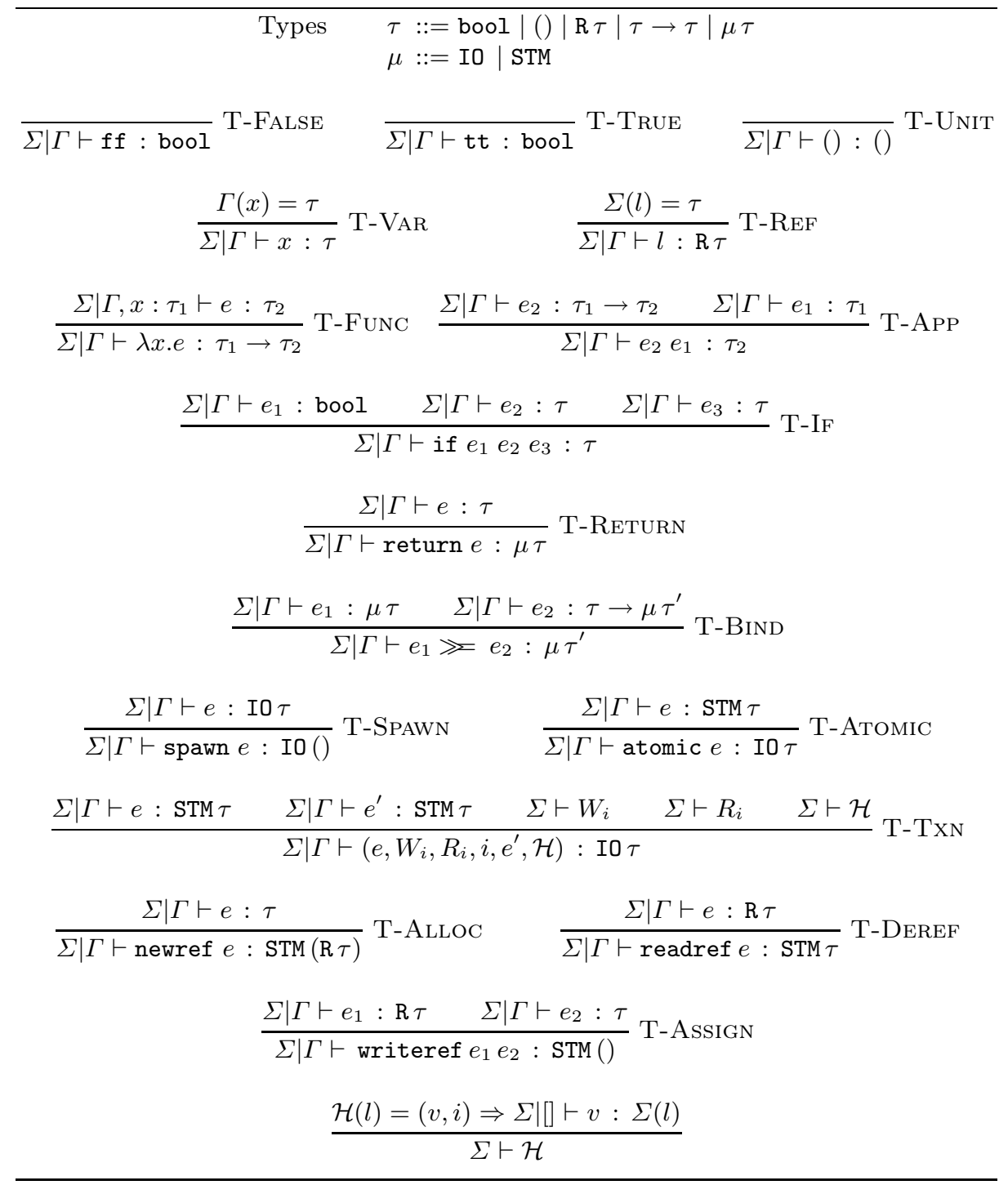

Fig. 2. Typing rules of $\Lambda_{S T M}$

\begin{tabular}{lll}
\hline$l$ & $\in$ Ref & \\
$\mathcal{P}$ & $\in$ Program & $=$ ThreadId $\rightarrow$ Exp \\
$T_{i} \quad \in$ Transaction & $=$ Exp $\times$ Store $\times$ Store $\times$ Id $\times$ Exp $\times$ Store \\
$\mathcal{H}, R_{i}, W_{i} \in$ Store & $=$ Ref $\rightarrow$ Val $\times$ Id \\
$\alpha$ & $\in$ Effect & $=\left\{\epsilon^{t}, s p^{t}, a t_{i}^{t}, a b_{i}^{t}, c o_{i}^{t}(\bar{l}), r_{i}^{t}(l), \epsilon_{i}^{t}\right\}$ \\
\hline
\end{tabular}

Fig. 3. State related definitions 
Evaluation contexts

$$
\begin{aligned}
\mathcal{E}::= & {[] e|v[]| \text { if }[] e e^{\prime} } \\
\mathcal{M}::= & \text { newref [] | readref [] } \mid \text { writeref [] } e \mid \text { writeref } v[] \\
& \mid \text { return [] | [] } \gg e
\end{aligned}
$$

Expression evaluation $\rightarrow$

$$
\begin{aligned}
(\lambda x . e) v & \rightarrow e[v / x] \\
\text { if tt } e e^{\prime} & \rightarrow e \\
\text { if ff } e e^{\prime} & \rightarrow e^{\prime} \\
\frac{e}{\mathcal{E}[e]} \rightarrow e^{\prime} &
\end{aligned}
$$

Monadic evaluation $\curvearrowright$

$$
\begin{array}{cc}
\frac{e \rightarrow e^{\prime}}{e \curvearrowright e^{\prime}} & \frac{m \curvearrowright m^{\prime}}{\mathcal{M}[m] \curvearrowright \mathcal{M}\left[m^{\prime}\right]}
\end{array}
$$

Fig. 4. Operational semantics: Local evaluation steps

pool maps thread identifiers to expressions to be evaluated concurrently. The execution of a program is represented by a labeled transition relation between program states.

A transaction in progress is represented by a tuple $\left(e, W_{i}, R_{i}, i, e^{\prime}, \mathcal{H}^{\prime}\right)$. It consists of the expression $e$ that is currently evaluated, the write set $W_{i}$ and the read set $R_{i}$ of the transaction, a (unique) transaction identifier $i$, a copy of the original transaction body $e^{\prime}$, and a copy $\mathcal{H}^{\prime}$ of the heap taken at the beginning of the transaction. The latter two store the relevant state at the beginning of a transaction to facilitate the consistency check and the abort operation.

A reference corresponds to a heap location. All stores (the heap, the read set, and the write set of a transaction) map a reference to a pair of a value and a transaction identifier. The transaction identifier specifies the transaction which committed or, in case of the write set, attempts to commit the value to the global store. $S(l)$ denotes the lookup operation of a reference $l$ in a heap $S$. It implies $l \in \operatorname{dom}(S)$. The store update operation $S[l \mapsto y]$ returns a store that is identical to $S$, except that it maps $l$ to $y$. For two stores $S_{1}$ and $S_{2}$, we write $S_{1}\left[S_{2}\right]$ for the updated version of $S_{1}$ with all entries of $S_{2}$.

Operations can have different effects $\alpha$ on the global state: the begin transaction $\left(a t_{i}^{t}\right)$, abort transaction $\left(a b_{i}^{t}\right)$, read reference $l\left(r_{i}^{t}(l)\right)$, and commit writing references $\bar{l}\left(c o_{i}^{t}(\bar{l})\right)$ indicating operations on the global shared heap, or empty effects $\left(\epsilon_{i}^{t}\right.$ or $\left.\epsilon^{t}\right)$, with $t$ a thread identifier, and $i$ a transaction id. The empty effects represent monadic reductions that occur outside a transaction (see top of Fig. 5).

The evaluation of a program with body $e$ starts in an initial state \langle\rangle$,\left\{t_{0} \mapsto e\right\}$ with an empty heap and a main thread $t_{0}$. A final state has the form $\mathcal{H},\left\{t_{0} \mapsto\right.$ 


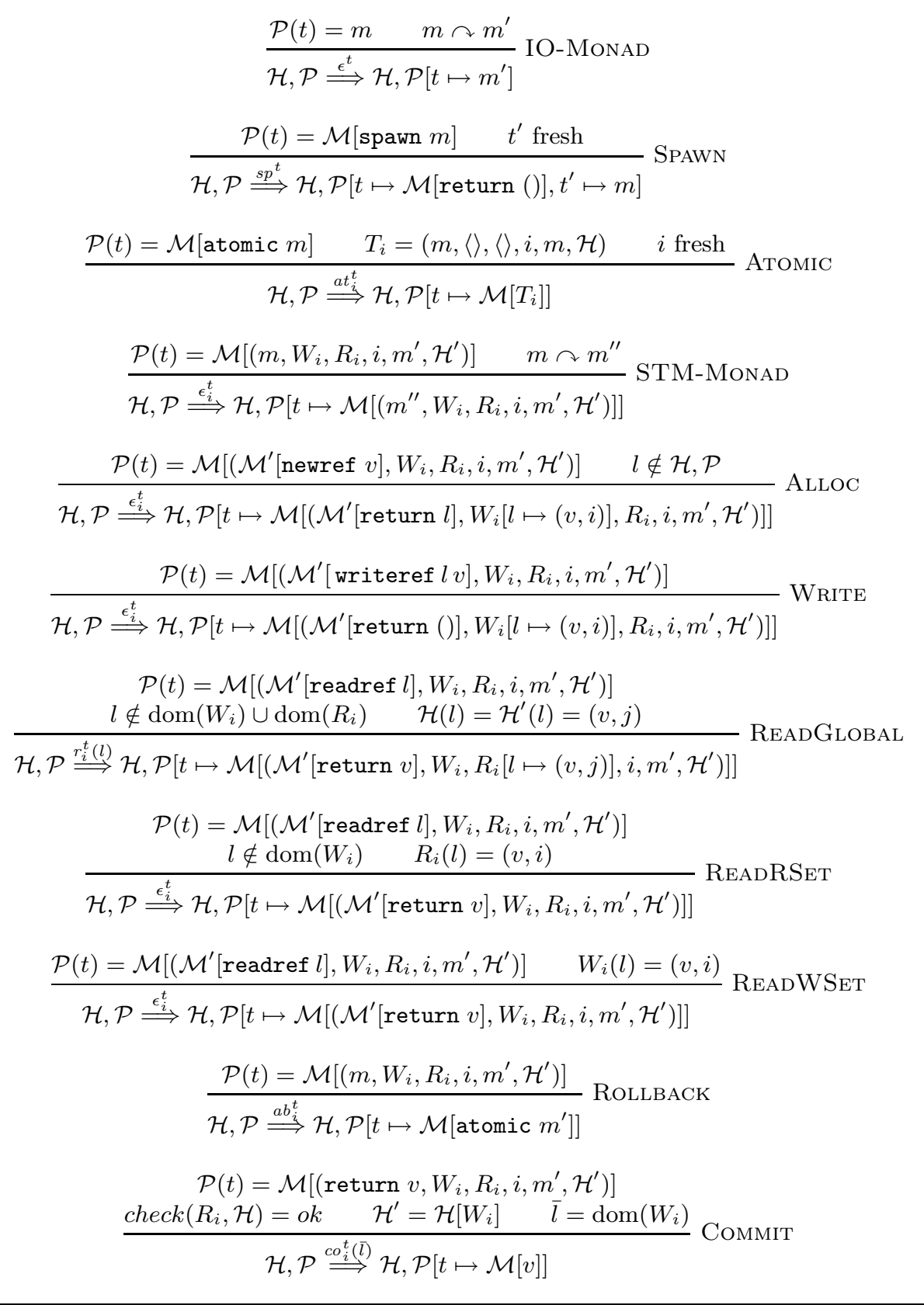

Fig. 5. Operational semantics: Global evaluation steps 


$$
\begin{aligned}
& \frac{\forall l \in \operatorname{dom}\left(R_{i}\right): R_{i}(l)=\mathcal{H}(l)}{\operatorname{check}\left(R_{i}, \mathcal{H}\right)=o k} \text { CHECK-OK } \\
& \frac{\exists l \in \operatorname{dom}\left(R_{i}\right): R_{i}(l) \neq \mathcal{H}(l)}{\operatorname{check}\left(R_{i}, \mathcal{H}\right)=\text { bad }} \text { CHECK-BAD }
\end{aligned}
$$

Fig. 6. Operational semantics: Helper relations

$\left.v_{0}, \ldots, t_{n} \mapsto v_{n}\right\}$. The rules in Figures 4 and 5 define the semantics of the language constructs. In Fig. 4, $\mathcal{E}[\bullet]$ denotes an evaluation context for an expression and $\mathcal{M}[\bullet]$ an evaluation context for monadic expressions. We write $m$ to indicate that an expression has monadic type.

The 10 monad is the top-level evaluation mode. Each reduction step $\stackrel{\alpha}{\Longrightarrow}$ chooses an expression from the thread pool $\mathcal{P}$. The non-determinism in this choice models an arbitrary scheduling of threads.

Spawning a thread (SPAwN) creates a new entry in the thread pool and returns unit in the parent thread.

An atomic expression at the top-level (Атоміс) creates a new transaction in progress with the expression to be evaluated, an empty read and write set, and a fresh transaction identifier (that has never been used before in a particular evaluation). Further, a copy of the expression $m$ is needed for possible rollbacks, and a copy of the current heap to mark the beginning of the transaction.

Any evaluation step that can take place outside a transaction, can also take place inside (STM-MONAD).

Allocation of a new reference (ALLOC) must check that the reference is not yet allocated in the heap. But it must also check that the reference is not yet allocated in any concurrently running transaction to avoid accidental overwrites when both transactions commit. This condition is indicated by $l \notin \mathcal{H}, \mathcal{P}$, eschewing a formal definition.

Write operations (WRITE) are straightforward. They just affect the local write set and store the value along with the current transaction identifier.

The read operation on references (READGLOBAL) needs to consult the global state. If a reference cannot be read from the local read or write set, it is accessed in the current global heap. To maintain the transaction's consistency, the read operation is successful only if the value has not been updated since the transaction's beginning. The value and transaction identifier as registered in the heap for this reference are then added to the read set and the value is returned to the transactional computation.

If a reference is present in the read set, but not in the write set, then its value is taken from the read set (READRSET).

If the reference is present in the write set, then its value is taken from the write set, without checking the read set (READWSET).

If none of the preceding three cases holds at a read, then the transaction aborts and rolls back via RoLLBACK by abandoning the transaction in progress 
and reinstalling the saved transaction body $m^{\prime}$ as an atomic block. In fact, this rule has no precondition so that a rollback may happen non-deterministically at any time during a transaction. This way, it is easy to extend our model with an explicit user abort or retry operation. Furthermore, this rule covers the abort both when reading fails as well as when the commit operation fails.

When committing (Сомміт), the heap is checked for updates to the references which are found in the transaction's read set since the start of the transaction. There are two cases:

The check is successful: None of the variables read by the transaction have been committed by another transaction in the meantime. Therefore, the transaction may publish its writes atomically to the shared heap and return to the IO monad.

The check fails: The only applicable rule is RoLLBACK. The transaction aborts and restarts.

Each of these reductions generates the appropriate effect label on the transition relation. Thus, each sequence of labeled reductions uniquely determines a sequence of labels, which we call the trace of the reduction sequence. Unlike other formalizations, the interleaving of transactions as well as the abort operations are visible in the trace.

Theorem 1 (Type soundness). The type system in Figure 0 is sound with respect to the operational semantics of $\Lambda_{S T M}$.

Proof. The proof is by establishing type preservation and progress in the usual way [15]. The proof of progress relies crucially on the use of the Rollback rule if the comparison of heap entries in READGLOBAL or Commit fails.

\section{Opacity}

Many TM systems implemented opacity before it was even defined. Examples systems are DSTM [9], McRT-STM [13], and TL2 [4]. Thus, opacity may be called a standard isolation property of STM. It states that any permitted interleaving of transactions must have an equivalent serialized execution. Furthermore, even aborting transactions are required to view memory locations only in a consistent way.

We can prove formally that the semantics for $\Lambda_{S T M}$ satisfies opacity. To this end, we give a definition for well-formedness of execution traces in terms of the effects they exhibit.

We then show that reordering certain evaluation steps leads to equivalent reductions sequences. Reductions are considered equivalent if each read operation returns the same value, each commit operation commits the same values, and each transaction's outcome (abort or commit) is the same. To see which reordering yields equivalent reductions, we define a notion of dependency on effects.

Finally, we show that all reduction sequences produced by the operational semantics are equivalent to some reduction sequence with a serial trace, up to 
the assignment of unique labels to the transactions. Without loss of generality, we only consider finite traces: for infinite traces, we would be able to establish our results for all finite prefixes.

\subsection{Well-Formedness}

The well-formedness of a trace depends largely on temporal relations between effects. We denote by $\alpha$ any effect from a trace $\bar{\alpha}, \alpha_{j}$ denotes the effect at index $j$ in the trace, $\alpha^{t}$ an effect from thread $t$, and $\alpha_{i}^{t}$ an effect from transaction $T_{i}$ in thread $t$.

Further, $\left.\bar{\alpha}\right|_{t}$ is the subset of all effects from thread $t$, and $\left.\bar{\alpha}\right|_{t, i}$ the subsets of all effects from transaction $i$ in thread $t$.

Definition 1 (Effect traces). For a trace $\bar{\alpha}$, a total order on the effects $\alpha \in \bar{\alpha}$ is defined by their position in the effect trace. For $i, j \in\{1, \ldots,|\bar{\alpha}|\}$ and $i \leq j$, we use the abbreviation

$$
\bar{\alpha} \vdash \alpha_{i}<\alpha_{j}
$$

to denote that an effect $\alpha_{i}$ is happening before $\alpha_{j}$ in an trace $\bar{\alpha}$. Similarly,

$$
\bar{\alpha} \vdash \bar{\beta}<\bar{\gamma}
$$

extends the relation to sets of effects if it holds pairwise for all elements.

Definition 2 (Well-formed traces). $A$ trace $\bar{\alpha}$ is the sequence $\bar{\alpha}=\alpha_{1} \ldots \alpha_{n}$ of effects $\alpha_{i} \in \boldsymbol{E f f e c t}, i \in 1, \ldots, n$. A trace $\bar{\alpha}$ is well-formed iff the following conditions hold:

- There is no effect for a thread before its spawn effect.

$$
\alpha^{t} \in \bar{\alpha} \Rightarrow s p^{t} \in \bar{\alpha} \wedge \bar{\alpha} \vdash s p^{t}<\alpha^{t}
$$

- There is no effect for a transaction $T_{i}$ before its atomic effect.

$$
\begin{gathered}
r_{i}^{t}(l) \in \bar{\alpha} \Rightarrow a t_{i}^{t} \in \bar{\alpha} \wedge \bar{\alpha} \vdash a t_{i}^{t}<r_{i}^{t}(l) \\
c o_{i}^{t}(\bar{l}) \in \bar{\alpha} \Rightarrow a t_{i}^{t} \in \bar{\alpha} \wedge \bar{\alpha} \vdash a t_{i}^{t}<c o_{i}^{t}(\bar{l}) \\
a b_{i}^{t} \in \bar{\alpha} \Rightarrow a t_{i}^{t} \in \bar{\alpha} \wedge \bar{\alpha} \vdash a t_{i}^{t}<a b_{i}^{t}
\end{gathered}
$$

- There is no read effect for a transaction $T_{i}$ after its commit or abort effect.

$$
\begin{gathered}
c o_{i}^{t}(\bar{l}) \in \bar{\alpha} \Rightarrow \forall r_{i}^{t}(l) \in \bar{\alpha}: \bar{\alpha} \vdash r_{i}^{t}(l)<c o_{i}^{t}(\bar{l}) \\
a b_{i}^{t} \in \bar{\alpha} \Rightarrow \forall r_{i}^{t}(l) \in \bar{\alpha}: \bar{\alpha} \vdash r_{i}^{t}(l)<a b_{t}^{i}
\end{gathered}
$$

- A transaction may have either a commit or an abort effect, but not both.

$$
\begin{aligned}
& \operatorname{co}_{i}^{t}(\bar{l}) \in \bar{\alpha} \Rightarrow a b_{i}^{t} \notin \bar{\alpha} \\
& a b_{i}^{t} \in \bar{\alpha} \Rightarrow c o_{i}^{t}(\bar{l}) \notin \bar{\alpha}
\end{aligned}
$$


- There are no non-transactional effects within a transaction.

$$
\epsilon^{t} \in \bar{\alpha} \Rightarrow \nexists i: \bar{\alpha} \vdash a t_{i}^{t}<\epsilon^{t}<c_{i}^{t}(\bar{l}) \text { or } \bar{\alpha} \vdash a t_{i}^{t}<\epsilon^{t}<a b_{i}^{t}
$$

- Transactional effects from the same thread do not interleave.

$$
\forall t \forall i \neq j:\left.\bar{\alpha} \vdash \bar{\alpha}\right|_{t, i}<\left.\bar{\alpha}\right|_{t, j} \text { or }\left.\bar{\alpha} \vdash \bar{\alpha}\right|_{t, j}<\left.\bar{\alpha}\right|_{t, i}
$$

Definition 3 (Pending transactions). A transaction $T_{i}$ is pending in a trace $\bar{\alpha}$ if it has neither a commit or an abort effect:

$$
a b_{i}^{t} \notin \bar{\alpha} \text { and } \operatorname{co}_{i}^{t}(\bar{l}) \notin \bar{\alpha}
$$

In contrast to other definitions of well-formed execution traces (e.g. [14]), we do not include the condition that the order of all reads and writes in the transaction is preserved in the effect traces. The operational semantics guarantees that each transaction is working on a consistent view of the shared memory as indexed by its time stamp. A read operation returns the last value written, either by another transaction which updated the global heap, or by the transaction itself in a local write step. Further, all write operations are published (i.e., made visible to other transactions) only after the successful commit. Therefore, the trace reflects the order of the globally visible effects of the read and write operations. The local reads and writes have no globally visible effect.

Lemma 1. All traces produced by type-correct programs are well-formed.

Proof. Type-correct programs allow only certain compositions of transactional phases. Effects are only produced when evaluating expressions in the STM monad. An $a t_{i}^{t}$ effect is only produced when entering the STM monad. All read effects are produced within the STM part, and the evaluation of a transactional expression finishes with either an $a b_{i}^{t}$ or $c o_{i}^{t}(\bar{l})$ effect.

Well-formedness of a trace relates the effects of one transaction to each other. Complementary, an isolation level defines a relation between the effects of all transactions that participate in a trace [2]. Serializability, for example, is one of these isolation levels.

Definition 4 (Serial traces). A well-formed trace $\bar{\alpha}$ is serial if for any two transactions $T_{i}$ and $T_{j}(i \neq j)$, all effects from $T_{i}$ occur before all effects from $T_{j}$, or vice versa:

$$
\forall i \neq j:\left.\bar{\alpha} \vdash \bar{\alpha}\right|_{t_{i}, i}<\left.\bar{\alpha}\right|_{t_{j}, j} \text { or }\left.\bar{\alpha} \vdash \bar{\alpha}\right|_{t_{j}, j}<\left.\bar{\alpha}\right|_{t_{i}, i}
$$

In contrast to other approaches, we do not exclude aborting or pending transactions in the definition for serial traces. Therefore, we actually model opaque traces.

Definition 5 (Control dependency). An effect $\alpha_{i}$ has a control dependency on an effect $\alpha_{j}, \alpha_{i} \triangleright_{c} \alpha_{j}$, iff they must occur in that order in any well-formed trace. A control dependency exists in the following cases: 


$$
\begin{aligned}
& -a t_{i}^{t} \triangleright_{c} r_{i}^{t}(l) \\
& -a t_{i}^{t} \triangleright_{c} c o_{i}^{t}(\bar{l}) \\
& -a t_{i}^{t} \triangleright_{c} a b_{i}^{t} \\
& -r_{i}^{t}(l) \triangleright_{c} a b_{i}^{t} \\
& -r_{i}^{t}(l) \triangleright_{c} c o_{i}^{t}(\bar{l})
\end{aligned}
$$

Definition 6 (Data dependency). An effect $\alpha_{i}$ has a data dependency on an effect $\alpha_{j}, \alpha_{i} \triangleright_{d} \alpha_{j}$, if they exhibit a write-read, read-write or write-write conflict. $A$ data dependency exists in the following cases where $i \neq j$ :

$$
\begin{aligned}
& -r_{i}^{t}(l) \triangleright_{d} c o_{j}^{t^{\prime}}(\bar{l}) \text { if } l \in \bar{l} \\
& -c_{i}^{t}(\bar{l}) \triangleright_{d} r_{j}^{t^{\prime}}(l) \text { if } l \in \bar{l} \\
& -c o_{i}^{t}(\bar{l}) \triangleright_{d} c o_{j}^{t^{\prime}}\left(\overline{l^{\prime}}\right) \text { if } \bar{l} \cap \bar{l}^{\prime} \neq \emptyset
\end{aligned}
$$

Definition 7 (Dependency). An effect $\alpha_{i}$ is dependent on an effect $\alpha_{j}, \alpha_{i} \triangleright$ $\alpha_{j}$, iff $\alpha_{i}$ is either control or data dependent on $\alpha_{j}$ and $\bar{\alpha} \vdash \alpha_{i}<\alpha_{j}$ in a trace $\bar{\alpha}$. Effects that are not dependent are called independent. A trace of effects $\bar{\alpha}$ is independent from another trace of effects $\bar{\alpha}^{\prime}$ iff all effects $\alpha_{m} \in \bar{\alpha}$ are independent from all effects $\alpha_{k} \in \bar{\alpha}^{\prime}$.

Definition 8 (Dependent transactions). A transaction $T_{i}$ is dependent on a transaction $T_{j}$ if $\alpha_{i} \triangleright \alpha_{j}$ for an effect $\alpha_{i}$ from $T_{i}$ and an effect $\alpha_{j}$ from $T_{j}$.

Definition 9 (Trace dependencies). Let $\bar{\alpha}$ be a well-formed trace. The trace dependencies $\Delta(\bar{\alpha})$ are defined as the set of all tuples of dependent effects in this trace:

$$
\Delta(\bar{\alpha})=\left\{\left(\alpha_{i}, \alpha_{j}\right) \mid \alpha_{i} \triangleright \alpha_{j}\right\}
$$

The trace dependencies impose a partial order on a trace. We are interested in equivalent traces that satisfy the same order and are permutations of each other. However, the order of trace items inside of every individual thread must not be changed. We call permutations that leave the relative ordering inside every thread unchanged admissible permutations. We prove that we can admissibly permute a trace such that it is serial, and that execution of this trace ends in the same program state.

In the scope of this paper, the term serializability actually refers to conflict serializability [14]. In contrast to view seriazability, which defines traces to be equivalent if the same data items are read and written, conflict serializability requires that equivalent traces preserve all dependencies.

Definition 10 (Equivalence of traces). A trace $\bar{\alpha}$ is equivalent to a trace $\bar{\beta}$ iff $\bar{\beta}$ is an admissibly permutation of $\bar{\alpha}$ and $\Delta(\bar{\alpha})=\Delta(\bar{\beta})$.

Definition 11 (Equivalence of program states). A program state $\mathcal{P}$ is equivalent to a program state $\mathcal{P}^{\prime}, \mathcal{P} \sim \mathcal{P}^{\prime}$ iff for all threads $i$ either $\mathcal{P}(i)=$ $\mathcal{P}^{\prime}(i)$ or $\mathcal{P}(i)=\mathcal{M}\left[\left(m_{1}, W_{i}, R_{i}, i, m_{2}, \mathcal{H}\right)\right], \mathcal{P}^{\prime}(i)=\mathcal{M}\left[\left(m_{1}, W_{i}, R_{i}, i, m_{2}, \mathcal{H}^{\prime}\right)\right]$ and $\left.\mathcal{H}\right|_{R_{i}}=\left.\mathcal{H}^{\prime}\right|_{R_{i}}$. 
Definition 12 (Equivalence of evaluation states). An evaluation state $\mathcal{H}, \mathcal{P}$ is equivalent to an evaluation state $\mathcal{H}^{\prime}, \mathcal{P}^{\prime}$ iff $\mathcal{H}=\mathcal{H}^{\prime}$ and $\mathcal{P} \sim \mathcal{P}^{\prime}$.

Lemma 2 (Permutation of reduction steps). Let $R$ be the two-step reduction

$$
\mathcal{H}, \mathcal{P} \stackrel{\alpha_{i}}{\Longrightarrow} \mathcal{H}_{0}, \mathcal{P}_{0} \stackrel{\alpha_{j}}{\Longrightarrow} \mathcal{H}^{\prime}, \mathcal{P}_{0}^{\prime}
$$

If $\alpha_{i}$ is independent from $\alpha_{j}$, then there exists an equivalent reduction sequence $R^{\prime}$ of the form

$$
\mathcal{H}, \mathcal{P} \stackrel{\alpha_{j}}{\Longrightarrow} \mathcal{H}_{1}, \mathcal{P}_{1} \stackrel{\alpha_{i}}{\Longrightarrow} \mathcal{H}^{\prime}, \mathcal{P}_{1}^{\prime}
$$

and $\mathcal{P}_{0}^{\prime} \sim \mathcal{P}_{1}^{\prime}$.

Proof. Case distinction on all independent effects.

Effect-free operations $\left(\alpha_{i}=\epsilon^{t}\right.$ or $\left.\alpha_{j}=\epsilon_{i}^{t}\right)$ are either pure or work on local (transactional) state. Therefore, these steps can get swapped with any operation while resulting in the same heap and thread pool. Reduction steps which result in an abort only modify the transactions' local state. The same holds for read operations. For commit effects, it holds that subsequent independent commit operations change disjoint parts of the global heap. The rules for inconsistency checks require that read and write sets of concurrently running transactions are disjoint in case of successful commit. Therefore reordering independent commit operations has no influence on the heap's final state, and produces equivalent program states.

In our semantics the begin of a transaction defines its relative order to other transactions. Yet, this order is only partial for transactions that perform their operations interleaved. In this case, they all only commit successfully if their operations do not conflict with each other. The following lemma shows that for these transactions, any relative order is permissive.

Lemma 3 (Permutation of committing transactions). Let $\bar{\alpha}$ be a wellformed trace with $\bar{\alpha}=\bar{\alpha}^{\prime} \operatorname{co}_{i}^{t}(\bar{l})$ and $\bar{\alpha}^{\prime}$ serial. Further, let $T_{j}$ be a transaction with $a t_{j}^{t_{j}} \in \bar{\alpha}$ and $\bar{\alpha} \vdash a t_{i}^{t_{i}}<a t_{j}^{t_{j}}<\operatorname{co}_{i}^{t_{i}}(\bar{l})$ and there does not exist a $k$ with $\bar{\alpha} \vdash a t_{i}^{t_{i}}<a t_{k}^{t_{k}}<a t_{j}^{t_{j}}$. Then $\bar{\alpha}$ is equivalent to a trace $\bar{\beta}$ with $\bar{\beta} \vdash \alpha_{j}<a t_{i}^{t_{i}}$ for all effects $\alpha_{j}$ of transaction $T_{j}$.

Proof. According to the restrictions, the trace must have the following structure:

$$
\bar{\alpha}=\alpha_{\text {pre }}, a t_{i}^{t_{i}}, \overline{r_{i}^{t_{i}}(l)}, a t_{j}^{t_{j}}, \overline{r_{j}^{t_{j}}(l)},\left(a b_{i}^{t_{i}} \mid c o_{i}^{t_{i}}(\bar{l})\right), \alpha_{\text {post }}
$$

There are no dependencies between $a t_{j}^{t_{j}}$ and any $r_{i}^{t_{i}}(l)$, or $a t_{j}^{t_{j}}$ and $a t_{i}^{t_{i}}$, or any $r_{i}^{t_{i}}(l)$ and any $r_{j}^{t_{j}}(l)$. By Lemma 3 this is therefore equivalent to trace

$$
\alpha_{\text {pre }}, a t_{j}^{t_{j}}, \overline{r_{j}^{t_{j}}(l)}, a t_{i}^{t_{i}}, \overline{r_{i}^{t_{i}}(l)},\left(a b_{i}^{t_{i}} \mid c o_{i}^{t_{i}}(\bar{l})\right), \alpha_{\text {post }}
$$

Case distinction on the status of $T_{j}$. 
- Case $a b_{j}^{t_{j}} \in \bar{\alpha}$ : There is no dependency between $a b_{j}^{t_{j}}$ and any effect of $T_{i}$, so by Lemma 2, the trace is equivalent to

$$
\alpha_{\text {pre }}, a t_{j}^{t_{j}}, \overline{r_{j}^{t_{j}}(l)}, a b_{j}^{t_{j}}, a t_{i}^{t_{i}}, \overline{r_{i}^{t_{i}}(l)}, \alpha_{\text {post }}
$$

- Case $\operatorname{co}_{j}^{t_{j}}(\bar{l}) \in \bar{\alpha}$ : Assume that $r_{i}^{t_{i}}(l) \triangleright \operatorname{co}_{j}^{t_{j}}(\bar{l})$. Then, the validation of the transaction $T_{i}$ in rule Commit would fail and $c o_{i}^{t_{i}}(\bar{l}) \notin \bar{\alpha}$ in contradiction to the assumption. Hence, $c o_{j}^{t_{j}}(\bar{l})$ is not dependent on any effect of $T_{i}$, and by Lemma 2, the trace is equivalent to

$$
\alpha_{\text {pre }}, a t_{j}^{t_{j}}, \overline{r_{j}^{t_{j}}(l)}, c o_{j}^{t_{j}}(\bar{l}), a t_{i}^{t_{i}}, \overline{r_{i}^{t_{i}}(l)}, \alpha_{\text {post }}
$$

- Case $T_{j}$ is pending: Then the trace $\bar{\alpha}$ is equivalent to

$$
\alpha_{\text {pre }}, a t_{j}^{t_{j}}, \overline{r_{j}^{t_{j}}(l)}, a t_{i}^{t_{i}}, \overline{r_{i}^{t_{i}}(l)}, \alpha_{\text {post }}
$$

End case distinction on the status of $T_{j}$.

In the remainder of this section, we identify which subsequences of a trace are not serial, and specify an algorithm that moves the effects to the appropriate place.

Lemma 4 (Conflicts). Let $\bar{\alpha}$ be a well-formed trace. Then $\bar{\alpha}$ is either serial, or there exists an $\alpha_{k}$ such that the prefix $\alpha_{1} \ldots \alpha_{k}$ is serial and

1. $\alpha_{k}$ and $\alpha_{k+1}$ are independent, or

2. $\alpha_{k}=r_{i}^{t_{i}}(l)$ and $\alpha_{k+1}=\operatorname{co}_{j}^{t_{j}}(\bar{l})$ with $l \in \bar{l}$.

Proof. We consider all possible combinations of effects which might occur in a well-formed trace. Cases that are left out lead violate well-formedness.

Case distinction on $\alpha_{i}$ and $\alpha_{k+1}$ where $i \neq j$.

- Case $\alpha_{k}=\epsilon^{t_{i}}$ or $\alpha_{k+1}=\epsilon^{t_{j}}$ : serial or independent.

- Case $\alpha_{k}=\epsilon_{j}^{t_{i}}$ or $\alpha_{k+1}=\epsilon_{j}^{t_{j}}$ : serial or independent.

- Case $\alpha_{k}=a t_{i}^{t_{i}}$ and $\alpha_{k+1}=a t_{j}^{t_{j}}$ : serial.

- Case $\alpha_{k}=a t_{i}^{t_{i}}$ and $\alpha_{k+1}=r_{i}^{t_{i}}(l)$ : serial.

- Case $\alpha_{k}=a t_{i}^{t_{i}}$ and $\alpha_{k+1}=r_{j}^{t_{j}}(l)$ : independent.

- Case $\alpha_{k}=a t_{i}^{t_{i}}$ and $\alpha_{k+1}=\operatorname{co}_{i}^{t_{i}}(\bar{l})$ : serial.

- Case $\alpha_{k}=a t_{i}^{t_{i}}$ and $\alpha_{k+1}=\operatorname{co}_{j}^{t_{j}}(\bar{l})$ : independent.

- Case $\alpha_{k}=a t_{i}^{t_{i}}$ and $\alpha_{k+1}=a b_{i}^{t_{i}}$ : serial.

- Case $\alpha_{k}=a t_{i}^{t_{i}}$ and $\alpha_{k+1}=a b_{j}^{t_{j}}$ : independent.

- Case $\alpha_{k}=r_{i}^{t_{i}}(l)$ and $\alpha_{k+1}=r_{j}^{t_{j}}\left(l^{\prime}\right)$ : independent.

- Case $\alpha_{k}=r_{i}^{t_{i}}(l)$ and $\alpha_{k+1}=r_{i}^{t_{i}}\left(l^{\prime}\right)$ : serial.

- Case $\alpha_{k}=r_{i}^{t_{i}}(l)$ and $\alpha_{k+1}=\operatorname{co}_{i}^{t_{i}}(\bar{l})$ : serial.

- Case $\alpha_{k}=r_{i}^{t_{i}}(l)$ and $\alpha_{k+1}=\operatorname{co}_{j}^{t_{j}}(\bar{l})$ : If $l \in \bar{l}$, then this is the second case in the lemma. Otherwise independent. 
- Case $\alpha_{k}=r_{i}^{t_{i}}(l)$ and $\alpha_{k+1}=a b_{i}^{t_{i}}$ : serial.

- Case $\alpha_{k}=r_{i}^{t_{i}}(l)$ and $\alpha_{k+1}=a b_{j}^{t_{j}}$ : independent.

- Case $\alpha_{k}=\operatorname{co}_{i}^{t_{i}}(\bar{l})$ and $\alpha_{k+1}=\operatorname{co}_{j}^{t_{j}}\left(\bar{l}^{\prime}\right)$ : According to the operational semantics, it must hold that $\bar{l} \cap \bar{l}^{\prime}=\emptyset$. Therefore, the effects are independent.

- Case $\alpha_{k}=\operatorname{co}_{i}^{t_{i}}(\bar{l})$ and $\alpha_{k+1}=a b_{j}^{t_{j}}$ : independent.

End case distinction on $\alpha_{i}$ and $\alpha_{k+1}$ where $i \neq j$.

For the proof of opacity, we define an algorithm which produces for a serializable trace an equivalent serial trace.

while $\bar{\alpha}$ is not serial do

choose $\alpha_{k}$ and $\alpha_{k+1}$ such that $\alpha_{1} \ldots \alpha_{k}$ is serial and $\alpha_{1} \ldots \alpha_{k+1}$ is not serial if $\alpha_{k}$ and $\alpha_{k+1}$ are independent then swap $\alpha_{k}$ with $\alpha_{k+1}$

else if $\alpha_{k}=r_{i}^{t_{i}}(l)$ and $\alpha_{k+1}=c o_{j}^{t_{j}}(\bar{l})$ with $l \in \bar{l}$ then

permute transactions in prefix such that prefix ends with transaction $j$

end if

end while

Fig. 7. Reordering transactions for opacity

The algorithm in Figure 7 has the following properties:

1. It terminates on all traces produced by a type-correct programs in $\Lambda_{S T M}$.

2. For any trace input of a type-correct program in $\Lambda_{S T M}$, it gives an equivalent serial trace.

We prove these properties in several steps.

Lemma 5 (Termination). The algorithm terminates on all traces of typecorrect programs in $\Lambda_{S T M}$.

Proof. We show that the algorithm only performs a finite number of swaps for each pair of effects. Further, for each iteration of the while loop, either a permutation or a swap is performed. The transaction permutations are performed at most $n$ ! times, where $n$ is the number of all transactions that participates in a trace. Let $m_{t}$ denote the number of effects that a transaction produces, and $m=\max m_{t}$. For every permutation, each pair of effects is swapped at most once $((m-1) !)$. As each trace consists only of a finite number of effects, the algorithm performs at most $n !(m-1)$ ! many swap operations.

Lemma 6 (Permutation). The output of the algorithm is a permutation of the input trace.

Proof. All operations on the trace are permutations of effects. Therefore, effects are neither removed from nor added to the input trace. 
Lemma 7 (Dependencies). The algorithm does not change any dependencies in the trace.

Proof. Effects are only swapped when they are independent or when permuting transactions. In the latter case, the dependencies in the trace are respected as is shown in Lemma 3 .

Lemma 8 (Correctness of the algorithm). The output of the algorithm is an equivalent serial trace.

Proof. By Lemmas [6] and [7] the output is equivalent to the input trace. By Lemma 5, the algorithm terminates on all traces from type-correct programs. In this case, the condition for entering the while loop is falsified, and therefore the trace is serial.

Theorem 2 (Opacity). Let $\mathcal{P}_{0}$ be a type-correct program. Further, let $R$ be a sequence of reductions

$$
\mathcal{H}_{0}, \mathcal{P}_{0} \stackrel{\alpha_{1}}{\Longrightarrow} \ldots \stackrel{\alpha_{n}}{\Longrightarrow} \mathcal{H}_{n}, \mathcal{P}_{n}
$$

Then there exists an equivalent sequence $R^{\prime}$ of the form

$$
\mathcal{H}_{0}, \mathcal{P}_{0} \stackrel{\alpha_{1}^{\prime}}{\Longrightarrow} \ldots \stackrel{\alpha_{n}^{\prime}}{\Longrightarrow} \mathcal{H}_{n}, \mathcal{P}_{n}^{\prime}
$$

such that $\bar{\alpha}\left(R^{\prime}\right)$ is serial.

Proof. We apply the algorithm for serialization of traces to the traces of $R$. Because the algorithm only requires the permutation of independent effects, by Lemma 2 the result is an equivalent reduction sequence with serial trace.

\section{Related Work}

Weikum and Vossen [14] include a comprehensive overview on theory and practice of transactional systems. Although their work is based on databases, the presented results relate to all transaction-based execution environments. They differentiate in detail between several notions of serializability, and give soundness proofs for all major commit protocols.

Jagannathan and coworkers [10] specify a formal system for transactions with nesting implemented by a versioning and a locking algorithm. They do not model aborts, but stuck executions are implicitly rolled back. They show that the presented algorithms implement serializability.

Abadi and coworkers [1] formalize the semantics of the Automatic Mutual Exclusion (AME) programming model. Similarly, Moore and Grossman [12, provide a formal model with small-step operational semantics for an impure functional language. Both works focus on the treatment of memory locations inside and outside of transactions, and in which cases the notion of weak and strong atomicity coincide. 
Doherty and coworkers [5] give a formalization for transactional memory in terms of an I/O automaton. Their specifications are of different granularities and aim to for machine-checked correctness proofs of implementations.

Opacity was introduced by Guerraoui and Kapalka [7] as a correctness criterion for transactional memory. They also show how opacity can be efficiently implemented for different relaxed memory models [6.

Our work is partially inspired by Lipton's work [11 on a reduction theory for proving properties of concurrent programs. His main idea was to identify certain statements that may be moved to the left or to the right in the trace of an interleaved execution. In particular, he establishes that lock acquisition can always be moved to the right over a statements executing in another thread, whereas lock release is a left mover. The commonality is that we are also reordering traces to prove isolation properties, but the difference is that we consider a transactional framework which also includes explicit transaction aborts.

\section{Conclusion and Outlook}

Transactional memory has evolved as a serious subdomain in concurrent programming. Yet, to this day only few formal models exists that give insight into properties of TM algorithms and its concrete semantics.

This paper presents the formalization of a TM algorithm with lazy update in terms of a monadic lambda calculus. The formal model incorporates all standard TM operations and introduces their abstractions as effects on the global state. The type system enforces strong atomicity semantics by restricting access to shared memory to the STM monad. Based on the traces, the semantics is shown to implement the isolation level of opacity.

We are currently extending the calculus with additional features like I/O actions and repair facilities for conflicts [3. So far, the small core calculus and its effect system facilitated the development of the enriched interface.

It is also possible to adapt the operational semantics such that it implements other isolation levels like snapshot isolation. The technique of reordering can be used in a similar fashion to transform execution traces to a canonical form of traces produced by snapshot isolation.

\section{References}

1. Abadi, M., Birrell, A., Harris, T., Isard, M.: Semantics of transactional memory and automatic mutual exclusion. In: POPL 2008: Proceedings of the 35th annual ACM SIGPLAN-SIGACT symposium on Principles of programming languages, pp. 63-74. ACM, New York (2008)

2. Berenson, H., Bernstein, P., Gray, J., Melton, J., O'Neil, E., O'Neil, P.: A critique of ansi sql isolation levels. In: SIGMOD 1995: Proceedings of the 1995 ACM SIGMOD International Conference on Management of Data, pp. 1-10. ACM, New York (1995)

3. Bieniusa, A., Middelkoop, A., Thiemann, P.: Brief announcement: Actions in the twilight - concurrent irrevocable transactions and inconsistency repair. In: Richa, A.W., Guerraoui, R. (eds.) PODC, pp. 71-72. ACM, New York (2010) 
4. Dice, D., Shalev, O., Shavit, N.: Transactional locking II. In: Dolev, S. (ed.) DISC 2006. LNCS, vol. 4167, pp. 194-208. Springer, Heidelberg (2006)

5. Doherty, S., Groves, L., Luchangco, V., Moir, M.: Towards formally specifying and verifying transactional memory. In: Proceedings of the RefineNet Workshop 2009 (REFINE 2009). Electronic Notes in Theoretical Computer Science (2009)

6. Guerraoui, R., Henzinger, T.A., Kapalka, M., Singh, V.: Transactions in the jungle. In: auf der Heide, F.M., Phillips, C.A. (eds.) SPAA, pp. 263-272. ACM, New York (2010)

7. Guerraoui, R., Kapalka, M.: On the correctness of transactional memory. In: Chatterjee, S., Scott, M.L. (eds.) PPOPP, pp. 175-184. ACM, New York (2008)

8. Harris, T., Marlow, S., Jones, S.P., Herlihy, M.: Composable memory transactions. In: Sixteenth ACM SIGPLAN Symposium on Principles and Practice of Parallel Programming, Chicago, IL, USA, pp. 48-60. ACM Press, New York (2005)

9. Herlihy, M., Luchangco, V., Moir, M., Scherer III, W.N.: Software transactional memory for dynamic-sized data structures. In: PODC 2003: Proceedings of the Twentysecond Annual Symposium on Principles of Distributed Computing, Boston, Massachusetts, pp. 92-101. ACM Press, New York (2003)

10. Jagannathan, S., Vitek, J., Welc, A., Hosking, A.: A transactional object calculus. Sci. Comput. Program 57(2), 164-186 (2005)

11. Lipton, R.J.: Reduction: A method of proving properties of parallel programs. Commun. ACM 18(12), 717-721 (1975)

12. Moore, K.F., Grossman, D.: High-level small-step operational semantics for transactions. In: POPL 2008: Proceedings of the 35th annual ACM SIGPLAN-SIGACT symposium on Principles of programming languages, pp. 51-62. ACM, New York (2008)

13. Saha, B., Adl-Tabatabai, A.-R., Hudson, R.L., Minh, C.C., Hertzberg, B.: McRTSTM: a high performance software transactional memory system for a multi-core runtime. In: PPoPP 2006: Proceedings of the 11th ACM SIGPLAN symposium on Principles and practice of parallel programming, New York, NY, USA, pp. 187-197 (2006)

14. Weikum, G., Vossen, G.: Transactional information systems: theory, algorithms, and the practice of concurrency control and recovery. Morgan Kaufmann Publishers Inc., San Francisco (2001)

15. Wright, A., Felleisen, M.: A syntactic approach to type soundness. Information and Computation 115(1), 38-94 (1994) 